\title{
PULMONARY VASCULAR COMPROMISE IN A CHILD WITH POSTPNEUMONECTOMY SYNDROME
}

\author{
Mark D. Rodefeld, MD, ${ }^{\text {a }}$ Frederic D. Wile, BA, ${ }^{a}$ Richard I. Whyte, MD, ${ }^{\text {a }}$ Paul T. Pitlick, MD, ${ }^{\mathrm{b}}$ and Michael D. Black, MD, \\ Stanford, Calif
}

Postpneumonectomy syndrome typically presents with airway obstruction. Tracheobronchial compression results from shifting of the mediastinal contents into the pneumonectomy space. It stands to reason that arterial or venous compromise could also occur; however, postpneumonectomy syndrome with vascular stenosis or obstruction has not been reported. Postpneumonectomy syndrome in infants and children is rare because the incidence of pneumonectomy in this age group is low. However, this population is at higher risk than adults. ${ }^{1,2}$ We report the case of a 1-year-old child with previous left pneumonectomy who had failure to thrive and a heart murmur resulting from critical pulmonary arterial stenosis.

Clinical summary. At 4 months of age, this infant boy had been treated in India for multiple episodes of respiratory distress. Congenital emphysema of the left upper lobe was diagnosed by chest radiograph, and resection was undertaken. For unclear reasons, a left pneumonectomy was performed. The patient recovered and his family subsequently relocated to the United States. On evaluation by his pediatrician, he had symptoms of failure to thrive and a new murmur was detected. Echocardiography demonstrated moderate to severe tricuspid regurgitation, moderately decreased right ventricular function, and stenosis of the right pulmonary artery. Right ventricular pressure was estimated to be nearly equal to systemic pres-

From the Departments of Cardiothoracic Surgery ${ }^{\mathrm{a}}$ and Pediatrics, ${ }^{\mathrm{b}}$ Stanford University School of Medicine, Stanford, Calif.

Received for publication Dec 20, 1999; accepted for publication Dec 23, 1999.

Address for reprints: Michael D. Black, MD, Department of Cardiothoracic Surgery, Falk Cardiovascular Research Center, Stanford University School of Medicine, Stanford, CA 943055407 (E-mail: michael.black@ stanford.edu).

J Thorac Cardiovasc Surg 2000;119:851-3.

Copyright (C) 2000 by The American Association for Thoracic Surgery.

$0022-5223 / 2000 \$ 12.00+0 \quad \mathbf{1 2 / 5 4 / 1 0 5 2 6 1}$

doi:10.1067/mtc. 2000.105261
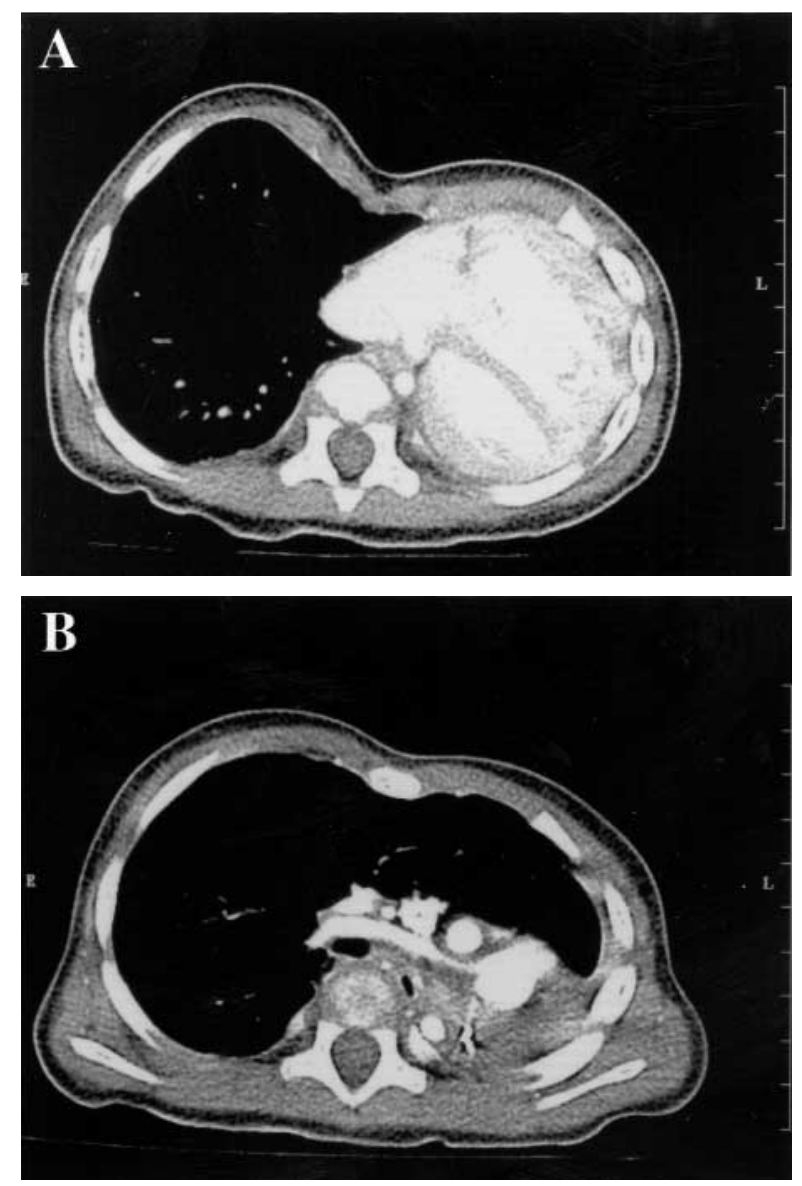

Fig 1. Preoperative electron beam computed tomography scan. A, Mediastinal shift with marked chest asymmetry. Cardiomediastinal structures fill the left hemithorax. B, Right pulmonary artery stenosis, most severe at its origin from the main pulmonary artery. Note anterior compression of the pulmonary artery by the aorta. The right bronchus is widely patent. 

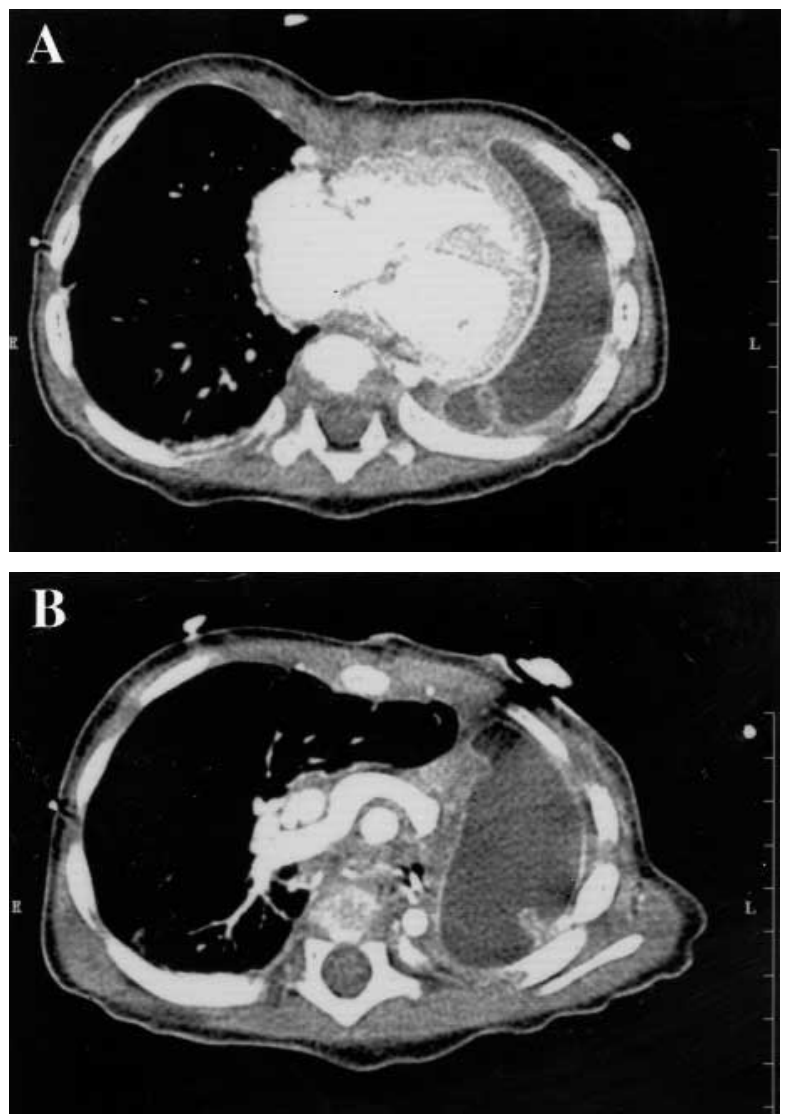

Fig 2. Postoperative computed tomographic scan. A, Repositioning of the mediastinum. Chest wall symmetry is substantially improved. B, Translocation of the pulmonary artery anterior to the aorta, which is now widely patent.

sure. The chest radiograph showed extreme mediastinal shift with displacement of the heart into the posterior left hemithorax. The right lung was hyperexpanded and filled the mediastinal space. Computed tomographic scanning confirmed stenosis of the right pulmonary artery, which was compressed by the vertebral column posteriorly and by the aorta anteriorly (Fig 1). The right bronchus was stretched, but widely patent.

Surgical repair was undertaken with two goals: relief of the pulmonary arterial stenosis and repositioning of the mediastinum to reduce the chance of future airway or vascular obstructive problems. Through a median sternotomy, the right lung was mobilized and retracted. Manipulation of the heart was poorly tolerated because of severe right ventricular hypertension, and cardiopulmonary bypass was urgently initiated. The heart was freed from the left pleural space and the pulmonary artery was dissected. The main pulmonary artery was dilated, whereas the right pulmonary artery was stenotic from its origin to the hilum with marked thickening of the vessel wall. After the mediastinum had been repositioned, it became clear that transposition of the pulmonary artery anterior to the ascending aorta would be hemodynamically advantageous. The pulmonary artery was divided and reconstructed anterior to the aorta, with a pulmonary homograft patch inserted anteriorly to complete the repair.

The volume of the left pleural space was estimated with saline solution, and a breast prosthesis $(80 \mathrm{~mL}$ saline solution in a $120-\mathrm{mL}$ capacity prosthesis) was inserted to reestablish correct anatomic configuration of the mediastinal structures. Cardiopulmonary bypass was discontinued and right ventricular pressure decreased to less than one half systemic pressure. Intraoperative transesophageal echocardiography showed resolution of tricuspid regurgitation. The patient was extubated the next day and recovered uneventfully. A followup computed tomographic scan demonstrated a widely patent pulmonary artery and significant improvement in mediastinal positioning and chest symmetry (Fig 2).

Discussion. Although the incidence is low, postpneumonectomy syndrome is more likely to develop in infants and young children than in adults..$^{1-3}$ Contributing factors unique to this age group include increased pliability and mobility of the mediastinal tissues, anatomic change as a result of somatic growth, and increased elasticity, compliance, and growth potential of the remaining lung. These allow for overdistention of the lung into the mediastinal space, thus potentiating the severity of mediastinal shift. Placement of a prosthesis into the pneumonectomy space at the time of resection has been recommended to avoid cardiorespiratory compromise associated with extreme mediastinal shift. An expandable prosthesis may be used with theoretical benefit related to growth. However, a conclusive advantage over a fixed prosthesis has not been demonstrated. A secondary benefit of prosthesis placement is preservation of chest wall symmetry, with long-term implications related to axial skeletal growth and quality of life.

Postpneumonectomy syndrome after left pneumonectomy is rare. ${ }^{4,5}$ Because of the natural leftward displacement of the heart, rightward mediastinal shift after right pneumonectomy results in more severe anatomic distortion. Surgical therapy is aimed at correction of the mediastinal shift and correction of associated airway or vascular compromise. Because the mediastinal structures may have become remodeled to accommodate the postpneumonectomy shift, overcorrection should be avoided as this may result in a different set of anatomic distortion problems. Furthermore, repair of airway or vascular problems should be addressed in the setting of the repositioned mediastinum. In this case, it became apparent that translocation of the pulmonary artery anterior to the aorta would be required after the heart had been relocated more centrally in the chest. A reconstruction posterior to the aorta before repositioning would likely have resulted in persistent stenosis.

Vascular compromise as a result of postpneumonectomy mediastinal shift has not been a reported consequence of the postpneumonectomy syndrome. If this child had been left untreated, right-sided heart failure and presumably death 
would have ensued. The arterial stenosis was related to several forces including compression of the artery between the vertebral column and aorta, longitudinal stretching, and pathologic thickening of the arterial wall. Whether the vascular thickening was due to anatomic deformation or an underlying vasculopathy is unknown. Percutaneous stenting was considered, but the severity of vessel wall thickening, the nonfocal nature of the stenosis, and absence of the left lung to provide adequate pulmonary blood flow during placement of a stent precluded its use. Regardless, repositioning of the mediastinum would not have been achieved. A child with significant growth potential such as in this case would presumably be at high risk for further pneumonectomy-related complications.

\section{REFERENCES}

1. Powell RW, Luck SR, Raffensperger VG. Pneumonectomy in infants and children: the use of a prosthesis to prevent mediastinal shift and its complications. J Pediatr Surg 1979;14:231-7.

2. Rasch DK, Grover FL, Schnapf BM, Clarke E, Polard TG. Right pneumonectomy syndrome in infancy treated with an expandable prosthesis. Ann Thorac Surg 1990;50:127-9.

3. Szarnicki R, Maurseth K, de Leval M, Stark J. Tracheal compression by the aortic arch following right pneumonectomy in infancy. Ann Thorac Surg 1978;25:231-5.

4. Quillin SP, Shackleford GD. Post-pneumonectomy syndrome after left lung resection. Radiology 1991;179:100-2.

5. Deslauriers J, Ferraro P. Late complications. In: Pearson FG, Deslauriers J. Thoracic surgery. 1st ed. New York: Churchill Livingstone; 1995. p. 775-6. 